\title{
A simple hypothesis of executive function
}

\section{Bruno Kopp*}

Cognitive Neurology, Technische Universität Braunschweig, Department of Neurology, Braunschweig Hospital, Germany

\section{Edited by:}

Hauke R. Heekeren, Freie

Universität Berlin, Germany

\section{Reviewed by:}

Juliana Yordanova, Institute of

Neurobiology, Bulgaria

Christopher Summerfield,

Oxford University, UK

*Correspondence:

Bruno Kopp, Department of Neurology, Braunschweig Hospital,

Salzdahlumer Str. 90, 38126

Braunschweig, Germany.

e-mail: b.kopp@klinikum-

braunschweig.de
Executive function is traditionally conceptualized as a set of abilities required to guide behavior toward goals. Here, an integrated theoretical framework for executive function is developed which has its roots in the notion of hierarchical mental models. Further following Duncan $(2010 a, b)$, executive function is construed as a hierarchical recursive system of test-operation-test-exit units (Miller et al., 1960). Importantly, it is shown that this framework can be used to model the main regional prefrontal syndromes, which are characterized by apathetic, disinhibited and dysexecutive cognition, and behavior, respectively. Implications of these considerations for the neuropsychological assessment of executive function are discussed.

Keywords: executive function, frontal lobes, inversion problem, hierarchical mental model, test-operation-test-exit (TOTE)

\section{PRELUDE}

The neuropsychological assessment of executive function is based on unsatisfactory experimental methods and clinical materials, even decades after the pioneering work by Luria (1966). One of the major difficulties of executive assessment is the paradox that was smartly described by Lezak (1995): the clinician has to structure a more or less formal situation "... in which patients can show whether and how well they make structure for themselves" (p. 651). Many of the examination techniques (Strauss et al., 2006) which are thought to be sensitive to executive, or frontal lobe, disorders (Wood and Grafman, 2003; Miller and Cummings, 2007; Banich, 2009; Levine and Craig, 2011; Stuss, 2011) clearly allow the subject insufficient room toward selfinduced problem structuring, decision making, and goal setting. Yet, it is immediately evident that these processes are main components of executive behavior, as revealed by Luria's, Lezak's, and many others' detailed descriptions of behavioral disturbances after frontal lobe damage.

The need for problem structuring is of particular importance in situations where executable behavioral and cognitive chunks are not readily available to the subject, or where their execution does not proximately lead to the aimed-for goal. Those situations enforce what Freud had called Probehandeln (mental action sampling) 100 years ago (Freud, 1911). Following Craig (1943), if the organism carries a mental model of "... external reality and its own possible actions within its head, it is able to try out various alternatives, conclude which is the best of them, react to future situations before they arise, utilize the knowledge of the past events in dealing with the present and the future, and in every day to react in a much fuller, safer, and more competent manner to the emergencies which face it" (p. 61). Some claims about the structure, the functionality, and the neuroanatomical distribution of mental models, including their potential relationships to frontal lobe damage, are presented below ${ }^{1}$.

\section{THE HIERARCHICAL STRUCTURE OF MENTAL MODELS}

Here, I develop the idea of hierarchically organized mental models which draws heavily on predictive coding theories of the perceptual system (Friston, 2002). Perception is considered as an active process of inference about underlying states of the world. Specifically, it is conjectured that perceptual analysis follows fundamentally an inversion purpose since it aims at retrieving the causes, i.e., the parameters of the generative mechanism, from the effects, i.e., the observed sensory data. In other words, when observing a sensory phenomenon directed by any parameter $\theta$, perceptual analysis allows inducing from these observations inferences about $\theta$ (Helmholtz, 1867). When the analysis generalizes over space and time, a prediction of future phenomena of similar nature becomes available, simply by exploiting the redundancy within the probability structure of $\theta$ (Barlow, 2001).

\footnotetext{
${ }^{1}$ The term "mental model" which is used here should not be confused with the mental model theory which is a theory of reasoning proposing that deductive and inductive reasoning depend on spatially organized mental models (Johnson-Laird, 1983). Here, mental models are merely internal representations of situations that can be derived from something the subject directly perceives in the environment.

Further, Tolman's (1948) term of a "cognitive map" signifies a similar concept. To quote from his famous rejection of behaviorism: "The stimuli, which are allowed in, are not connected by just simple one-to-one switches to the outgoing responses. Rather, the incoming impulses are usually worked over and elaborated in the central control room into a tentative, cognitive-like map of the environment. And it is this tentative map, indicating routes and paths and environmental relationships, which finally determines what responses, if any, the animal will finally release" (Tolman, 1948, p. 192).
} 
Suppose a human observer is exposed to a rapidly changing environment where multiple states coexist (either sequentially through time or simultaneously through space). When confronted with this kind of fast-changing environments, the observer should ideally infer the state probabilities, implying that he or she represents the probability structure of the environment hierarchically. In order to do so, he or she needs to learn a state hyper-parameter $\tau$, and to differentiate between state-specific parameters (i.e., $\theta_{1}$ and $\theta_{2}$ in the case of two coexisting states; see Figure 1). The capability to form such hierarchical generative models of fast-changing environments will greatly enhance the predictive performance of the observer.

Note that according to this analysis, inference, and prediction are operations on the same hierarchical data structure. This view is compatible with predictive coding theories of cortical function (Friston, 2002). Predictive coding theories posit that the perceptual system is structured as hierarchically organized generative models with increasingly more general nodes (parameters) at higher levels. Further, predictive coding theories propose that an internal representation of the world (i.e., the mental model) generates predictions that are compared with stimulus-driven activity to calculate the residual error between the prediction and the sensory evidence. The residual error is then used to update the internal representation of the world so as to minimize the residual error imposed by future stimuli.

Another implication of this analysis lies in the fact that the brain remembers the past to predict the future (Bar, 2011). The close relationship between experience and memory, as stored in mental models, on one hand and prediction and anticipation on the other is paralleled by the recently discovered fact that episodic memory and episodic future thinking share a common neural substrate in cerebral cortex (Schacter et al., 2008). This led researchers to ask whether time in the brain is a onedirectional variable, as psychologically experienced, or whether the brain is actually capable to travel back and forth through time (Suddendorf et al., 2009).

This analysis portrayed the perceptual system as being composed of hierarchically organized generative models. In what follows I will conceptualize executive function, required to guide

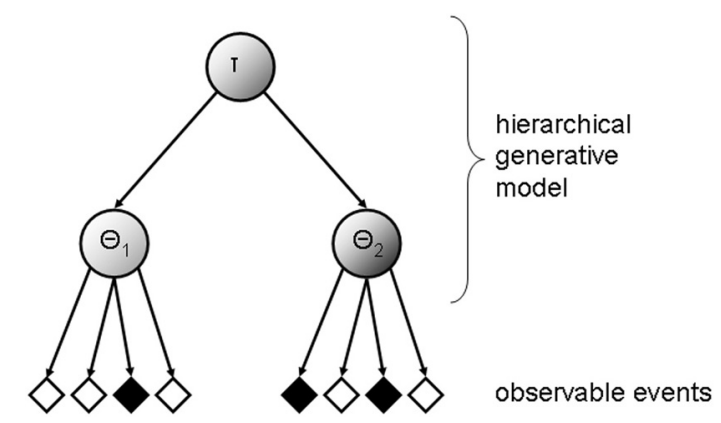

FIGURE 1 | Illustration of a hierarchical generative model of observed sensory data. The inferred probability structure of underlying states of the world is hierarchically represented, with $\tau$ signifying a state

hyper-parameter, $\theta_{1}$ and $\theta_{2}$, denoting dissociable state-specific parameters (just two levels of the hierarchy are drawn for illustration). intentional behavior toward goals, as a hierarchically organized test-retest system, an idea which draws heavily on Miller et al. (1960). Note that, while the analysis moves from the perceptual system to the executive system, it retains the idea of hierarchically organized mental models. Specifically, recursive activation of selfterminating operating units lies at the core of the suggested model of executive function.

\section{THE BEHAVIORAL FUNCTIONALITY OF MENTAL MODELS}

Frontal lobe damage often disrupts the patient's capacity for intentional behavior, it leads in many cases to the appearance of stimulus-bound behavior and inflexible responding, and it sometimes limits the ability to initiate behavior at all (Duffy and Campbell, 2001). The fundamental conjecture of this paper is that these behavioral syndromes which result from frontal lobe damage may be considered as observable effects of deficient mental models, as detailed below.

Lashley (1951) had argued long ago that behavior is hierarchically structured, and Miller et al. (1960) introduced the "test-operate-test-exit" (TOTE) unit to cognitive psychology just a few years later. When selected, a TOTE unit would test a particular environmental state and, if a specified condition was not met, would execute a specific operation until the condition became true. Miller et al. (1960) suggested that all kinds of behavior are controlled by TOTE units and used the everyday example of hammering a nail. The unit consists of a test-operator [Is the nail down? (Is the goal state achieved?)], an operation [hammering (and return to test)], and an exit-operator [to be executed if the goal state has been achieved (see test-operator)]. Crucially, the operation of a TOTE unit could be to select another TOTE unit, allowing for hierarchical structuring based on recursive processing (Corballis, 2011), i.e., by running a series of nested TOTE units. Details of the idea of recursive activation of self-terminating operating units are illustrated in Figure 2. Inspection of Figure 2 reveals that mental models may be considered as hierarchical structures of self-terminating operating units, each capable of activating otherwise comparable units at sub-ordinate levels of the hierarchical structure (Botvinick, 2008; Duncan, 2010a,b). Notice that the multiple-demand system framework (Duncan, 2010a,b) provides partly similar accounts of the crucial features of internal models guiding sequential behavior in complex situations. Specifically, both views share the idea of hierarchically organized internal models (see also Miller et al., 1960). The view which is presented here is oriented toward the manifold behavioral disturbances which usually follow frontal lobe lesions and which were shortly foreshadowed above, whereas Duncan's model (Duncan, 2010a,b) is focused more on the neurocognitive mechanisms of complex problem solving and (fluid) intelligence.

The formation of such recursive mental TOTE models constitutes the cognitive basis for executive behavior, this being characterized by the constant occurrence of intentional, goal-directed, and well-organized units of behavior (Shallice, 1988). The capability to model situations hierarchically in one's own mind and to achieve recursively derived goals of actions is a general purpose mechanism (Duncan, $2010 a, b)$ that can be applied to many seemingly different situations and with regard to a multitude of input and 


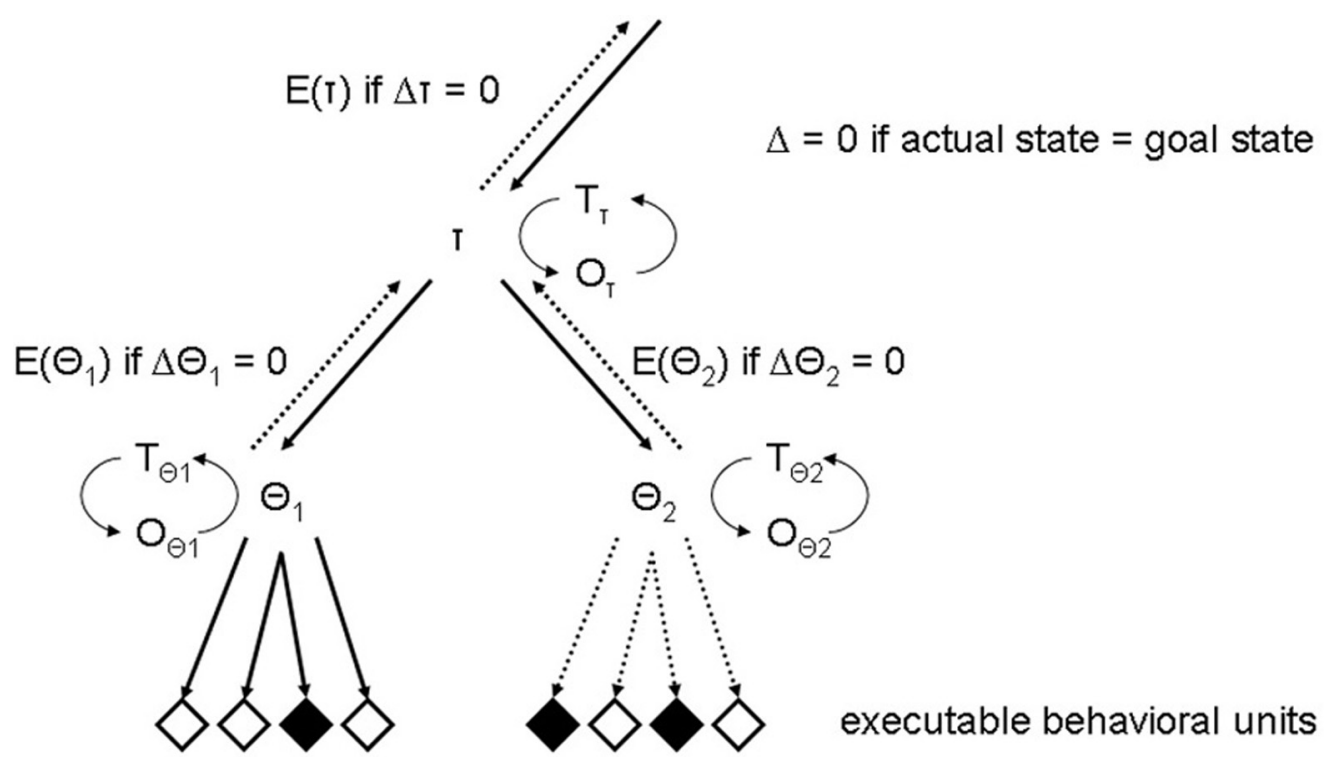

FIGURE 2 | Illustration of a recursive mental TOTE model capable of controlling intentional behavior (just two levels are drawn for illustration). TOTE units are self-terminating operating units, each capable of activating otherwise comparable units at sub-ordinate levels of the hierarchical structure (top-down arrows). The exit-operator of the active TOTE unit passes control of operations to the super-ordinate TOTE unit (bottom-up arrows), once the self-terminating condition is reached. Each TOTE unit is comprises three operators ( $T$, test; $\mathrm{O}$, operation; $\mathrm{E}$, exit). Activated test-operation-retest loops are running until the actual state matches the goal state. The exit-operator is executed (i.e., $E())$ whenever the self-terminating condition is reached (i.e., $\Delta=0$ ), so that control of operations is passed to the super-ordinate unit. Only TOTE units at the lowest level of the hierarchical structure are capable of activating executable behavioral units, whereas units at the hyper-planes are solely capable of activating units at sub-ordinate levels of the hierarchical structure. In this example, a hyper-unit, labeled $\tau$, activates a unit at the lowest level of the hierarchical structure, labeled $\theta_{1}$, which in turn activates executable behavioral units (solid arrows). In contrast, the connections between the un-activated unit $\theta_{2}$ at the lowest level and executable behavioral units are not active (dashed arrows). Switching activation states requires executing the exit-operator of unit $\theta_{1}$, once its self-terminating condition has been reached, i.e., $E\left(\theta_{1}\right)$ if $\Delta \theta_{1}=0$, and the activation of unit $\theta_{2}$ by the hyper-unit $\tau$. By way of this analysis, the current hypothesis of executive function offers a framework for understanding the effects of prefrontal lesions on behavioral performance in task switching paradigms (Robbins, 2007; Shallice et al., 2008; Nyhus and Barceló, 2009; Kopp and Wessel, 2011). output modalities. It gives the organism an adaptive advantage, specifically when confronted with the need to behave appropriately in complex and/or fast-changing environments (e.g., Behrens et al., 2007; Ribas-Fernandes et al., 2011)².

\section{MENTAL MODELS AND FRONTAL LOBE DAMAGE}

Duffy and Campbell (2001) described three distinct prefrontal syndromes, the apathetic, disinhibited, and dysexecutive types, each associated with dysfunction in one of the three major prefrontal cortical regions (i.e., medial, orbital, and lateral, respectively; see Kouneiher et al., 2009, as well as Rushworth et al., 2011, for discussion of the behavioral functionality of these prefrontal regions). As a start, I present a scheme for representing these

\footnotetext{
${ }^{2}$ The formation of hierarchically structured recursive mental models, the abilities to sample mental actions and to predict their environmental consequences in order to select the best alternative for the true course of actions introduces the teleological idea of final causes into human behavior. To put it in Ernst Mayr's words: "Intentional, purposeful human behavior is, almost by definition, teleological .... If teleological means anything, it means goaldirected .... Goal-directed behavior is extremely widespread in the organic world .... The occurrence of goal-directed processes is perhaps the most characteristic feature of the world of living systems" (E. Mayr; downloaded 3-15-2011 from http://faculty.washington.edu/lynnhank/Mayr3.pdf).
}

distinct behavioral syndromes of prefrontal dysfunction within the framework of recursive mental TOTE models.

The medial prefrontal syndrome is easiest to conceptualize. The common behavioral symptom of apathy, or in the most severe cases of akinetic mutism, could be viewed as resulting from a disconnection between otherwise intact recursive mental TOTE models and executable behavioral units (see Figure 3A). Consequently, patients with superior medial damage are usually slower on tasks that require speeded responses, a behavioral deficit which has been described as a failure of energization, that is, the process of initiating any response (Stuss, 2011). Since the structure of the recursive mental TOTE models is left intact, the failure of energization should present itself as a pure motivational deficit.

Disinhibited behavior is the cardinal feature of the orbital prefrontal syndrome which might express itself as increased distractibility on complex tasks and as environmental dependency in social situations (Lhermitte, 1986; Lhermitte et al., 1986; see Fellows, 2011, for a more recent conceptualization of the behavioral functionality of the orbitofrontal cortex). In essence then, responding by these patients is stimulus-bound rather than guided by the recursive mental TOTE models (see Figure 3B). Thus, exogenously activated executable behavioral units may be carried out, without being activated by the 

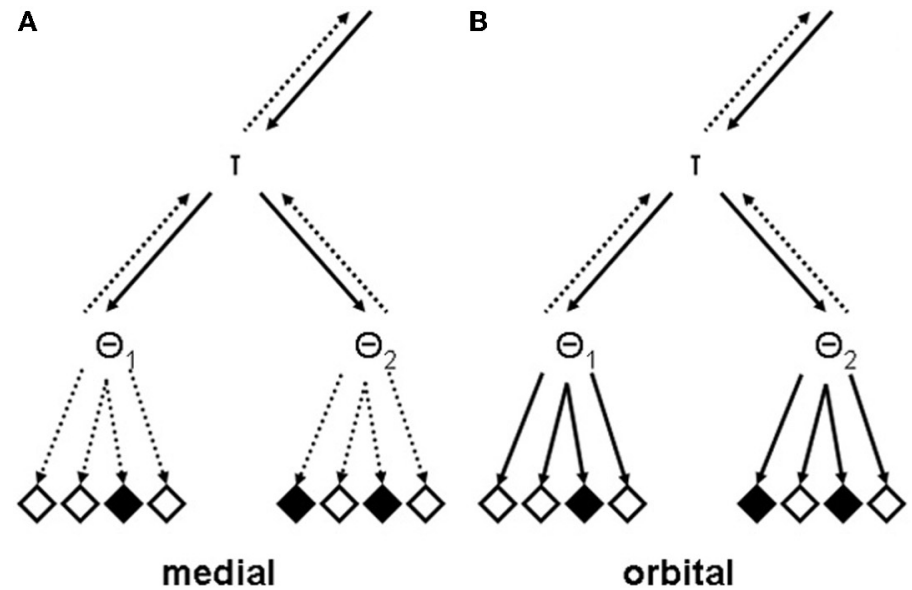

$\because$

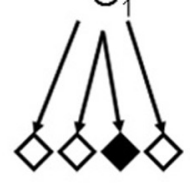

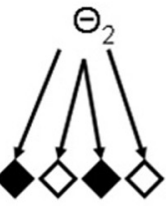

orbital
C

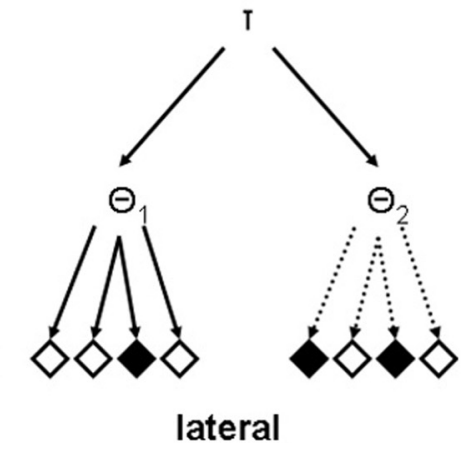

FIGURE 3 | Analytically lesioned recursive mental TOTE models.

(A) A potential lesion that might underlie the medial syndrome with predominantly apathetic symptoms. Note that the connections between both units at the lowest level of the hierarchical structure, $\theta_{1}$ and $\theta_{2}$, and executable behavioral units are inactive (dashed arrows). (B) A potential lesion that might underlie the orbital syndrome with predominantly disorganized symptoms. Note that the connections between both units at the lowest level of the hierarchical structure, $\theta_{1}$ and $\theta_{2}$, and executable behavioral units are active (solid arrows). (C) Potential lesions that might underlie the lateral syndrome with predominantly dysexecutive symptoms. There are two putative lesion effects: First, they may impair the functionality of the exit-operators, as detailed in the text. Second, they may destroy units at any level of the hyper-planes (this is solely adumbrated here by cancelling topmost arrows). It should be noticed that the various types of lesion effects are not mutually exclusive. They may occur together in one patient, in various degrees, depending on the nature, the locus, and the extension of his or her brain lesion (Duffy and Campbell, 2001; Stuss, 2011). endogenously structured system for controlling overt actions. By way of these direct routes from stimuli to actions, the recursive mental TOTE models loose their ability to exert control over behavior.

The lateral prefrontal syndrome has always been the least tractable of all prefrontal syndromes, due to the complexities of the cognitive and behavioral disturbances that are characteristic for this syndrome. Yet, within the framework which I presented above, the lateral syndrome starts to loose its chimeric character. Figure 3C illustrates the effects that lateral prefrontal lesions might have on the recursive mental TOTE models. Notice that primarily transitions from sub-ordinate to super-ordinate levels of the hierarchy became impossible in the lesioned model. A brief look back to Figure 2 reveals that these bottom-up transitions are produced by the exit-operators within the recursive mental TOTE models. Thus, the hypothesis states simply that lateral prefrontal lesions impair the exit-operators of the recursive mental TOTE models.

The impairment of bottom-up transitions might be brought into being by two dissociable processes. First, lateral prefrontal lesions might impair the functionality of the exit-operators, rendering them unable to actually produce the required transitions. Second, lateral prefrontal lesions (Petrides, 2005) and also inferior medial prefrontal lesions (Ridderinkhof et al., 2004) might impair state monitoring which is required for the decision whether or not a specified condition for self-termination has been met as a result of an elapsed operation of the unit, rendering it unable to actually self-terminate its activation. Yet, in both cases, the lesioned model will produce inflexible responding and perseverative behavior, by leaving the organism unable to switch back and forth between units of the recursive mental TOTE models (as detailed in the caption of Figure 2).
Impaired exit-operators and impaired state monitoring render the behavior of patients with lateral prefrontal lesions perseverative, i.e., they are expected to show a tendency to repeat previous actions over and over, albeit these are not appropriate any more. To illustrate let us take the example depicted in Figure 3C. There are three TOTE units, one at the higher level $(\tau)$, representing the goal state of the problem at hand, and two at the lower $\left(\theta_{1}, \theta_{2}\right)$, representing subgoals. If $\tau$ passed control to $\theta_{1}$ (which, however, cannot be exited any more, due to the effects of lateral prefrontal lesions), its assigned behavioral units will be repeatedly executed, whereas the solution to the problem at hand requires to pass control first to $\theta_{1}$ and afterwards to $\theta_{2}$, via $\tau$. In other words, these patients will appear stuck-in-set, unable (a) to flexibly adapt to actual requirements and (b) to solve problems smoothly. Whereas patients suffering from impaired exit-operators may be aware of the inappropriateness of their perseverative tendencies, patients suffering from impaired state monitoring may be characterized by a lack of awareness of their perseverative behavior, mainly because these patients should be generally unable to compare the results of actions with the intended goals.

However, there remains still another possibility how lateral prefrontal lesions might impair the functionality of recursive mental TOTE models. Instead of impairing exit-operators, these lesions may destroy units at super-ordinate levels of the hierarchically structured models (as depicted in Figure 3C). An organism will have difficulty whenever it is confronted with problems that require contributions of the lesioned units for their solution. Specifically, eminently novel, complex, and abstract problems may fall into this range of tasks (Goel, 2010). Apart from deficient problem solving skills, a general shift from abstract to more concrete attitudes for concept formation and reasoning may result from such structural lesions of recursive 
mental TOTE models, as for example evidenced by disturbed categorical performance on sorting tasks (Nyhus and Barceló, 2009) ${ }^{3}$. Finally, deficient decision making and planning for the long-term future (Damasio and Anderson, 2003) may constitute another corollary of these structural lesions of recursive mental TOTE models.

Further, Fuster (2008) proposed in his influential book that the prefrontal cortex is organized hierarchically, such that control is supported in progressively rostral regions as decisions are made at more abstract levels (see also Koechlin et al., 2003; Badre, 2008; Botvinick, 2008). Consistent with the concept of hierarchically arrayed levels of control, recent neuroimaging studies have repeatedly demonstrated differences in functional activation along the rostro-caudal axis of lateral frontal cortex, ranging from lateral frontal polar cortex to premotor cortex, such that more anterior regions were associated with progressively more abstract action control (Badre et al., 2009, 2010). This body of neuroscientific knowledge may be reconciled with the current hypothesis by the assumption that the hyper-planes of the recursive mental TOTE models are hierarchically arrayed in the prefrontal cortex, such that progressively rostral regions support progressively higher levels.

${ }^{3}$ Eling et al. (2008) trace the otherwise lost history of sorting tasks from the studies of Ach on the psychology of thinking, via the work of Goldstein and Gelb on brain lesioned patients around 1920 and subsequent developments, up to the actual design of the Wisconsin Card Sorting Test (Heaton et al., 1993) by Harlow, Grant, and Berg back in the $40 \mathrm{~s}$ of the twentieth century.

\section{REFERENCES}

Badre, D. (2008). Cognitive control, hierarchy, and the rostro-caudal organization of the frontal lobes. Trends Cogn. Sci. 12, 193-200.

Badre, D., Hoffman, J., Cooney, J. W., and D'Esposito, M. (2009). Hierarchical cognitive control deficits following damage to the human frontal lobe. Nat. Neurosci. 12, 515-522.

Badre, D., Kayser, A. S., and D'Esposito, M. (2010). Frontal cortex and the discovery of abstract action rules. Neuron 66, 315-326.

Banich, M. T. (2009). Executive function. The search for an integrated account. Curr. Dir. Psychol. Sci. 18, 89-94.

Bar, M. (2011). Predictions in the Brain: Using Our Past to Generate a Future. Oxford: Oxford University Press.

Barlow, H. (2001). Redundancy reduction revisited. Netw. Comput. Neural Syst. 12, 241-253.

Behrens, T. E. J., Woolrich, M. W., Walton, M. E., and Rushworth, M. F. S. (2007). Learning the value of information in an uncertain world. Nat. Neurosci. 10, 1214-1221.

Bishara, A. J., Kruschke, J. K., Stout, J. C., Bechara, A., McCabe, D. P., and
Busemeyer, J. R. (2010). Sequential learning models for the Wisconsin card sort task: assessing processes in substance dependent individuals. J. Math. Psychol. 54, 5-13.

Botvinick, M. M. (2008). Hierarchical models of behavior and prefrontal function. Trends Cogn. Sci. 12, 201-208.

Corballis, M. C. (2011). The Recursive Mind: The Origins of Human Language, Thought, and Civilization. Princeton, NJ: Princeton University Press.

Craig, K. J. W. (1943). The Nature of Explanation (Reprinted 1952). Cambridge: Cambridge University Press.

Damasio, A. R., and Anderson, S. W. (2003). "The frontal lobes," in Clinical Neuropsychology, 4th Edn, eds K. M. Heilman and E. Valenstein (New York, NY: Oxford University Press), 431-446.

Duffy, J. D., and Campbell, J. J. (2001). "Regional prefrontal syndromes," in The Frontal Lobes and Psychiatric Illness, eds D. P. Salloway, P. F. Malloy, and J. D. Duffy (Washington, DC: American Psychiatric Publishing Inc), 113-123.

\section{SEQUELAE}

There are a number of corollaries of the considerations which I presented here. First, a reduced output on fluency tasks, impaired organization and monitoring of material to be remembered, and of the subject's own responses, are among the major cognitive deficits after frontal lobe damage (Milner and Petrides, 1984). Neuropsychological assessment of executive functioning should in the future more explicitly address the structuring of stimulus materials and behavioral responses in formal examinations, giving the subject sufficient room to think of the situations they are confronted with, thereby explicitly offering them the opportunity to establish mental models for themselves. An appropriate conceptual as well as psychometric refinement of examination techniques would be most appreciated. Second, with the progress of those assessment instruments, new questions for empirical research could be posed. There is a remarkable paucity of theoretical investigations into the elements of executive function in the neuropsychological literature (but see Hazy et al., 2007; Hinz et al., 2009; Bishara et al., 2010; Shallice and Cooper, 2011). Consequently, experimental methods and clinical materials suffer from the lack of theoretical understanding of executive function. Model-based approaches are needed in future studies of the cognitive neuropsychology of executive function. Moving beyond traditional disciplinary boundaries would greatly facilitate the achievement of that goal. The work presented in this paper should be considered an initial step in the direction of getting formal with mental models, i.e., the cognitive basis of executive function.

Duncan, J. (2010a). How Intelligence Happens. New Haven, CT: Yale University Press.

Duncan, J. (2010b). The multipledemand (MD) system of the primate brain: mental programs for intelligent behaviour. Trends Cogn Sci. 14, 172-179.

Eling, P., Derckx, K., and Maes, R. (2008). On the historical and conceptual background of the Wisconsin card sorting test. Brain Cogn. 67, 247-253.

Fellows, L. K. (2011). Orbitofronta contributions to value-based decision making: evidence from humans with frontal lobe damage. Ann. N.Y Acad. Sci. 1239, 51-58.

Freud, S. (1911). Formulierungen über die zwei Prinzipien des psychischen Geschehens. Gesammelte Werke, Band VIII. Frankfurt a.M.: Fischer, 230-238.

Friston, K. (2002). Beyond phrenology: what can neuroimaging tell us about distributed circuitry? Annu. Rev. Neurosci. 25, 221-250.

Fuster, J. M. (2008). The Prefrontal Cortex, 4th Edn. San Diego, CA: Academic Press.

Goel, V. (2010). Neural basis of thinking: laboratory problems versus real-world problems. WIREs Cogn. Sci. 1, 613-621.

Hazy, T. E., Frank, M. J., and O'Reilly, R. C. (2007). Towards an executive without a homunculus: computational models of the prefrontal cortex/basal ganglia system. Philos. Trans. R. Soc. Lond. B Biol. Sci. 362, 1601-1613.

Heaton, R. K., Chelune, G. J., Talley, J. L., Kay, G. G., and Curtis, G. (1993). Wisconsin Card Sorting Test Manual: Revised and Expanded. Odessa, FL: Psychological Assessment Resources.

Helmholtz, H. von. (1867). Handbuch der Physiologischen Optik. Leipzig. Voss.

Hinz, A. M., Kostov, A., Kneißl, F., Sürer, F., and Danek, A. (2009). A mathematical model and a computer tool for the tower of Hanoi and tower of London puzzles. Inf. Sci. 179, 2934-2947.

Johnson-Laird, P. N. (1983). Mental Models: Toward a New Science of Language, Inference, and Consciousness. Cambridge: Cambridge University Press.

Koechlin, E., Ody, C., and Kouneiher, F. (2003). The architecture of cognitive control in the human 
prefrontal cortex. Science 302, Luria, A. R. (1966). Higher Cortical 1181-1185.

Kopp, B., and Wessel, K. (2011). Dissociating proactively and retroactively cued task switching: a route towards neuropsychological analyses of cognitive control. Neuropsychol. Trends 10, 55-70.

Kouneiher, F., Charron, S., and Koechlin, E. (2009). Motivation and cognitive control in the human prefrontal cortex. Nat. Neurosci. 12, 939-945.

Lashley, K. S. (1951). "The problem of serial order in behavior," in Cerebral Mechanisms in Behavior: The Hixon Symposium, ed L. A. Jeffress (Oxford: Wiley), 112-146.

Levine, B., and Craig, F. I. M. (2011). Mind and the Frontal Lobes: Cognition, Behavior, and Brain Imaging. New York, NY: Oxford University Press.

Lezak, M. D. (1995). Neuropsychological Assessment, 3rd Edn. New York, NY: Oxford University Press.

Lhermitte, F. (1986). Human autonomy and the frontal lobes. Part II: patient behavior in complex and social situations: the "environmental dependency syndrome”. Ann. Neurol. 19, 335-343.

Lhermitte, F., Pillon, B., and Serdaru, M. (1986). Human autonomy and the frontal lobes. Part I: Imitation and utilization behavior: a neuropsychological study of 75 patients. Ann. Neurol. 19, 326-334. Functions in Man. New York, NY: Basic Books.

Miller, B. L., and Cummings, J. L. (2007). The Human Frontal Lobes: Functions and Disorders, 2nd Edn. New York, NY: Guilford Press.

Miller, G. A., Galanter, E., and Pribram, K. A. (1960). Plans and the Structure of Behavior. New York, NY: Holt, Rinehart and Winston.

Milner, B., and Petrides, M. (1984). Behavioural effects of frontal-lobe lesions in man. Trends Neurosci. 7, 403-407.

Nyhus, E., and Barceló, F. (2009). The Wisconsin card sorting test and the cognitive assessment of prefrontal executive functions: a critical update. Brain Cogn. 71, 437-451.

Petrides, M. (2005). Lateral prefrontal cortex: architectonic and functional organization. Philos. Trans. R. Soc. Lond. B Biol. Sci. 360, 781-795.

Ribas-Fernandes, J. J. F., Solway, A., Diuk, C., McGuire, J. T., Barto, A. G., Niv, Y., and Botvinick, M. M. (2011). A neural signature of hierarchical reinforcement learning. Neuron 71, 370-379.

Ridderinkhof, K. R., Ullsperger, M., Crone, E. A., and Nieuwenhuis, S. (2004). The role of medial frontal cortex in cognitive control. Science 306, 443-447.

Robbins, T. W. (2007). Shifting and stopping: fronto-striatal substrates, neurochemical modulation and clinical implications. Philos. Trans. R. Soc. Lond. B Biol. Sci. 362, 917-932.

Rushworth, M. F. S., Noonan, M.-A P., Boorman, E. D., Walton, M. E., and Behrens, T. E. (2011). Frontal cortex and reward-guided learning and decision-making. Neuron 70 , 1054-1069.

Schacter, D. L., Addis, D. R., and Buckner, R. L. (2008). Episodic simulation of future events: concepts, data, and applications. Ann. N.Y. Acad. Sci. 1124, 39-60.

Shallice, T. (1988). From Neuropsychology to Mental Structure. Cambridge: Cambridge University Press.

Shallice, T., and Cooper, R. P. (2011). The Organisation of Mind. Oxford: Oxford University Press.

Shallice, T., Stuss, D. T., Picton, T. W. Alexander, M. P., and Gillingham, S. (2008). Mapping task switching in frontal cortex through neuropsychological group studies. Front. Neurosci. 2, 79-85. doi: 10.3389/neuro.01.013.2008

Strauss, E., Sherman, E. M. S., and Spreen, O. (2006). A Compendium of Neuropsychological Tests: Administration, Norms and Commentary, 3rd Edn. New York, NY: Oxford University Press.

Stuss, D. T. (2011). Functions of the frontal lobes: relation to executive functions. J. Int. Neuropsychol. Soc. 17, 759-765.
Suddendorf, T., Addis, D. R., and Corballis, M. C. (2009). Mental time travel and the shaping of the human mind. Philos. Trans. R. Soc. Lond. B Biol. Sci. 364, 1317-1324.

Tolman, E. C. (1948). Cognitive maps in rats and men. Psychol. Rev. 55, 189-208.

Wood, J. N., and Grafman, J. (2003). Human prefrontal cortex: processing and representational perspectives. Nat. Rev. Neurosci. 4, 139-147.

Conflict of Interest Statement: The author declares that the research was conducted in the absence of any commercial or financial relationships that could be construed as a potential conflict of interest.

Received: 06 February 2012; accepted: 18 May 2012; published online: 05 June 2012.

Citation: Kopp B (2012) A simple hypothesis of executive function. Front. Hum. Neurosci. 6:159. doi: 10.3389/ fnhum.2012.00159

Copyright (c) 2012 Kopp. This is an open-access article distributed under the terms of the Creative Commons Attribution Non Commercial License, which permits non-commercial use, distribution, and reproduction in other forums, provided the original authors and source are credited. 